\title{
Systems thinking, systems doing
}

\author{
The multidimensional problems of food require integrated solutions. Yet, there is a lack of clarity on the \\ operationalization of systems thinking in research. This is a major challenge for those working towards the Food \\ Systems Summit.
}

I igh-level questions of applied research are inherently multidimensional. However, they are often framed in a reductionist way, and methods used to address them can fall short of capturing their complexity. Though food as a research field is undoubtedly interdisciplinary, with human and planetary health at its centre, several of the teleconnections between agriculture, trade, the natural landscape, climate change and human behaviour are frequently not taken into account or poorly represented. Defining a nutritionally ideal diet without understanding what it takes to produce it sustainably, or designing agricultural systems solely aimed at supplying more calories, is bound to generate single-issue conclusions of limited practical relevance.

The United Nations Sustainable Development Goals (SDGs) have helped scientists move towards systemic research by providing a framework, common goals and a standardized language, as well as revealing linkages that may previously have been opaque. The World in 2050 an initiative launched by the Sustainable Development Solutions Network, the International Institute for Applied Systems Analysis and the Stockholm Resilience Centre - illustrates the unifying role of the SDGs in exploring synergies and trade-offs permeating the simultaneous pursuit of hunger, poverty, energy, growth and environment goals. Studies such as the one by Liu et al. ${ }^{1}$ used the SDGs as a guiding principle to operationalize and standardize multidisciplinary research. Other examples exist, and it is now imperative that systems are centre-stage during the inception and design of studies - and that SDGs are never a footnote or an afterthought.

The stipulation of funders and project leaders to consider SDGs does not always translate into a 'systems mindset'. This must be established early on, at the stage of problem framing - particularly if targets and policy recommendations are to be consistent across local and global scales. Moreover, while a diversity of methods is important for validation of outcomes and generation of complementary insights, a shared understanding of what it means to 'account for a given nexus' is paramount for effective communication among researchers, policy makers and society at large. It could be argued that the solution is even more foundational and the mindset for a systems approach should be imbued and consolidated during the education of food scientists, before pen is even put to paper on that first research proposal.

It is, nonetheless, impossible to be all-encompassing. Limitations exist in tools and data. For instance, adding nutrient-specific modelling constraints can make land use or crop production calculations more complicated, and datasets that are globally consistent tend to be too coarse for detailed assessments. Uncertainties are inevitable when it comes to future trends in technology deployment, societal behaviour, price elasticities, and so many determinants of food availability, access and utilization. The point, however, is that we can do more with what is already available. Computational power has improved immensely, experience has been accumulated, and the amount of data that can be compiled - especially in the face of the latest big-data developments - is substantially larger. Altogether, these elements open up opportunities to tackle intricate real-world problems more accurately and inclusively, and to broaden the scope of analysis without compromising its depth. Better data, in particular, can reveal parameter sensitivities and the strength of systemic interactions, thus reducing the need to pre-determine such relationships and associated path dependencies.

It is noteworthy that several statistical and computational models now look at a much larger set of variables, as is the fact that these models have been increasingly used to explore development pathways in food production and consumption under a range of scenarios and in a more holistic way. Efforts undertaken by specific groups to be more systemic, including AgMIP, the EAT-Lancet Commission and the Food Systems Dashboard, can help trigger a broader transition in the research community.

Research methods and frameworks are the lens through which we look at a problem. The way they are built or applied will determine the space of solutions that can be explored. As our world is increasingly interlinked and socio-environmental problems approach a point of collapse, the risks of disengagement from systems thinking - or an illusory shift towards it are not to be underestimated.

Published online: 11 November 2020 https://doi.org/10.1038/s43016-020-00190-9

References

1. Liu, J. et al. Nat. Sustain. 1, 466-476 (2018). 